\title{
Óptimos técnicos para la producción de leche y carne en el sistema bovino de doble propósito del trópico mexicano
}

\author{
Yuridia Bautista Martínez a \\ José Antonio Espinosa García ${ }^{\text {b* }}$ \\ José Guadalupe Herrera Haro ${ }^{c}$ \\ Francisco Ernesto Martínez Castañeda ${ }^{\mathrm{d}}$ \\ Humberto Vaquera Huerta ${ }^{c}$ \\ Benigno Estrada Drouaillet ${ }^{a}$ \\ Lorenzo Danilo Granados Rivera ${ }^{\mathrm{e}}$
}

${ }^{a}$ Universidad Autónoma de Tamaulipas. Facultad de Medicina Veterinaria y Zootecnia. Carretera Mante Km 5. 87000. Ciudad Victoria Tamaulipas, México.

${ }^{\mathrm{b}}$ Instituto Nacional de Investigaciones Forestales Agrícolas y Pecuarias (INIFAP). Centro Nacional de Investigación Disciplinaria en Fisiología y Mejoramiento Animal, Ajuchitlan Querétaro, México.

${ }^{\mathrm{c}}$ Colegio de Postgraduados, Campus Montecillo. Texcoco. Edo. México, México.

${ }^{\text {d } U n i v e r s i d a d ~ A u t o ́ n o m a ~ d e l ~ E s t a d o ~ d e ~ M e ́ x i c o . ~ T o l u c a . ~ E d o . ~ M e ́ x i c o, ~ M e ́ x i c o . ~}$

${ }^{\text {e}}$ Campo Experimental General Terán, INIFAP. General Terán. Nuevo León, México.

*Autor de correspondencia: espinosa.jose@ inifap.gob.mx

\section{Resumen:}

El objetivo de este estudio fue estimar y analizar los resultados obtenidos de una función de producción de leche y carne en unidades de producción del sistema de doble propósito (DP). 
Los datos se obtuvieron a través de encuestas mensuales, donde se registró información de producción de leche, carne, ingresos y egresos económicos, durante 12 meses. Las funciones se estimaron por el método indirecto de regresión lineal con datos transformados para una función Cobb-Douglas. La función para leche mostró que los insumos alimento y vacas explican el $91 \%$ de la producción, con coeficientes de elasticidad de 0.34 y 0.5 , productos marginales de 0.75 y 892.2 con valores de $\$ 4.03$ y $\$ 4,800.2$ respectivamente. Los insumos utilizados para la producción de leche se encuentran en la etapa II de producción con rendimientos marginales decrecientes, mientras que para la producción de carne los insumos alimento y vacas explican el $72 \%$ de la producción, con coeficientes de elasticidades de producción de -0.20 y 1.11 respectivamente; el alimento se ubicó en la etapa III de producción con rendimientos marginales negativos, y el insumo vacas se encontró en la etapa I con rendimientos marginales crecientes. La suma de los coeficientes de ambas funciones que fueron 0.92 y 0.91 por ser menores a uno, tienen rendimientos decrecientes a escala. El nivel óptimo técnico de producción fue de 488.97 L diarios y 10 becerros al año. Los insumos para producción de leche se están utilizando de forma racional, pero se debe evaluar la cantidad de alimento utilizada para producción de carne, ya que refleja que es sobre utilizado.

Palabras clave: Cobb-Douglas, Elasticidad, Producto marginal, Rendimientos a escala y Óptimo técnico.

Recibido:07/06/2018

Aceptado:29/09/2018

\section{Introducción}

En México en el año 2017 se produjeron 11,808 millones de litros de leche y se importaron 3,728 millones, para cubrir la demanda. Mientras que la producción de carne de bovino fue de 1,845 miles de toneladas y se importaron 136 miles para cubrir la demanda ${ }^{(1)}$, lo anterior indica que México no es autosuficiente en estos alimentos, es por esto que deben analizarse de manera cuantitativa la eficiencia de los sistemas especializado, semi especializado, doble propósito y familiar de producción de leche y carne para optimizar el uso de sus recursos. El sistema bovino de doble propósito se desarrolla principalmente en regiones tropicales ${ }^{(2)}$, se caracteriza por producir leche mediante el ordeño y carne a través de la venta de becerros al destete $^{(3)}$. Una de sus principales ventajas es que se reducen costos de alimentación, ya que la mayor parte de ésta es a base del pastoreo, y en algunos casos hay una suplementación para las vacas en producción al momento de la ordeña ${ }^{(2)}$. 
Un aspecto importante del sistema bovino de doble propósito, es el análisis de las unidades de producción que lo integran, en términos de eficiencia, a través del uso adecuado de los insumos utilizados para la producción ${ }^{(4)}$. Para estudiar el funcionamiento de estos sistemas y con el objetivo de expresar las relaciones causa-efecto se han desarrollado metodologías paramétricas que se fundamentan en la estimación de funciones de producción, en las cuales se muestra la relación entre las distintas cantidades de insumos y cantidades de producto obtenido, además de asociar a cada insumo con el nivel máximo de producción por periodo. Esto resulta importante ya que permite formular estrategias de desarrollo productivo para una región en particular.

Dado que la producción de leche y carne influyen factores fisiológicos y no fisiológicos como la cantidad y calidad de alimento, forrajes, tamaño del hato, estación del año, el número y la etapa de lactancia, etc ${ }^{(5)}$. Es importante conocer los factores que mejor explican la producción de leche y carne para seleccionar la cantidad de insumo que se debe utilizar y hacer un uso óptimo de éste ${ }^{(6)}$. Uno de los modelos para la determinación de las funciones de producción comúnmente utilizado en ganadería es la función Cobb-Douglas, aplicada para estimar la producción de leche y carne en diferentes sistemas y regiones de México ${ }^{(7,8,9)}$. De acuerdo a las propiedades de una función tipo Cobb- Douglas y a la teoría de la producción, se pueden calcular indicadores como las elasticidades de producción, que es el cambio porcentual de la cantidad producida en relación al cambio porcentual de los niveles de los insumos ${ }^{(10)}$; los rendimientos marginales, que muestra la disminución o aumento de un producto al añadir un insumo, y dependiendo de su comportamiento: crecientes, decrecientes, cero o negativos, indica si el insumo analizado está en la etapa I, II y III respectivamente en una función de producción clásica. También permite conocer el tipo de rendimientos a escala de la unidad de producción pecuaria, que explica cómo se comporta la producción en respuesta a una variación proporcional y simultánea de todos los insumos, estos pueden ser crecientes, constantes o decrecientes. Por otra parte, con el precio de venta de los insumos se estima el producto marginal, que es la variación en la cantidad producida, debido al incremento unitario de alguno de los insumos productivos, manteniendo los demás constantes y también el valor del producto marginal, que es el ingreso adicional que obtiene la empresa pecuaria, por una unidad de insumo adicional ${ }^{(11)}$. Esta información es útil para auxiliar a asesores económicos del sector pecuario, a los extensionistas encargados de asesorar a los productores y a los productores mismos, para realizar un uso racional de los recursos, además de decidir si le es conveniente aumentar o disminuir la cantidad de insumos utilizada en el proceso de producción para alcanzar mayores beneficios económicos. Por lo tanto, el objetivo de este estudio fue estimar y analizar los resultados obtenidos de una función de producción de leche y carne en unidades de producción representativas del sistema de doble propósito (DP) en el trópico mexicano, para conocer los insumos que tienen mayor influencia en la producción y conocer si son utilizados de forma racional, a través del cálculo del óptimo técnico sujeta al precio de los insumos y precio de venta de la leche y carne. 


\title{
Material y métodos
}

\begin{abstract}
Área de estudio
El estudio se realizó en unidades de producción de tres estados de la república mexicana representativas del trópico de Mexicano, en donde el sistema de producción que predomina es el de DP; Tabasco localizado $\left(17^{\circ} 51^{\prime} \mathrm{N}\right.$ y $\left.93^{\circ} 23^{\prime} \mathrm{O}\right)$ a $2 \mathrm{msnm}$ con un clima cálido húmedo y abundantes lluvias en verano, temperatura media anual de $26.4{ }^{\circ} \mathrm{C}$ y precipitación media mensual de $190.85 \mathrm{~mm}$; Chiapas (1541'12" $\mathrm{N}$ y 93¹2’33” O) a $57 \mathrm{msnm}$ con un clima cálido sub húmedo, temperatura media anual de $28^{\circ} \mathrm{C}$, precipitación media mensual de $80 \mathrm{~mm}$ y Sinaloa, $\left(23^{\circ} 14^{\prime} 29^{\prime \prime} \mathrm{N}\right.$ y $106^{\circ} 24^{\prime} 35^{\prime}$ O O) a $10 \mathrm{msnm}$ con un clima tropical semi húmedo, temperatura media anual de $26.0{ }^{\circ} \mathrm{C}$, precipitación media mensual de 63 $\mathrm{mm}^{(12)}$. Las unidades de producción se caracterizaron por utilizar cruzas de razas Bos Indicus y Bos Taurus con alimentación basada en pastoreo extensivo y complementación con dietas comerciales balanceadas con base a la energía neta de lactancia y $17 \%$ de proteína al momento de la ordeña para vacas altas y medianas productoras, con un promedio de 39 vacas en producción, que son ordeñadas una vez al día con el apoyo del becerro para estimular la bajada de la leche, tres cuartos de la ubre eran ordeñado para la venta y un cuarto se dejó al becerro para su alimentación. Mientras que la producción de carne en las unidades de producción consistió en la venta de becerros destetados al alcanzar un peso promedio de 160 $\mathrm{kg}$.
\end{abstract}

\section{Clasificación de insumos y variables productivas}

Los datos se obtuvieron a través de encuestas mensuales a los productores durante el periodo de junio del 2012 a julio del 2013, en 30 unidades de producción, 10 por cada estado. Las UP se seleccionaron por muestreo aleatorio irrestricto, de las UP inscritas en las asociaciones ganaderas locales y cooperantes con la adopción de cédulas de registro de información sobre estructura del hato, uso de la tierra, ingresos por venta de leche y carne, además de los egresos por la compra de insumos para la producción. Las variables utilizadas para la función de producción se basaron en las recomendaciones para la estimación de problemas cuando la empresa genera productos múltiples como la ganadería y agricultura. Y cuando éstas pueden cambiar sustancialmente de una región a otra, tales como cantidad de alimento concentrado, ganado, mano de obra y combustibles ${ }^{(13)}$. Por tanto, se capturaron las siguientes variables: producción total de leche en el año (litros), número total de becerros producidos en el año, cantidad de alimento concentrado utilizado en la unidad de producción en un año $(\mathrm{kg})$, número de vacas en producción, hectáreas en pastoreo, forraje conservado utilizado en la unidad de producción en un año $(\mathrm{kg})$, mano de obra permanente y temporal (jornales), número de sementales, costos por insumos de operación (electricidad, gasolina, diésel). 


\section{Análisis de datos}

Para estimar las funciones de producción de leche y carne, se utilizó el método indirecto para obtener una función tipo Cobb-Douglas, el cual consiste en una regresión lineal con los datos originales transformados a logaritmos neperianos, de las variables dependientes e independientes $^{(6)}$.

Una vez trasformadas cada una de las variables, se seleccionó el modelo que mejor explica la producción de leche y carne con el procedimiento STEPWISE de SAS. Este procedimiento inicia determinando la matriz de correlación simple, con base a los valores de correlación, se incluye en el modelo la variable independiente $\left(\mathrm{X}_{\mathrm{i}}\right)$ que tiene la correlación más alta con la variable respuesta $\left(\mathrm{Y}_{\mathrm{i}}\right)$. Empleando los coeficientes de correlación parcial $\left(\mathrm{R}^{2}\right)$, se selecciona la siguiente variable a incluir en el modelo. A cada paso examina la contribución de cada variable al modelo, usando como criterio la prueba de F parcial, por lo tanto, en cada etapa todas las variables son examinadas por su contribución única al modelo, y aquellas que no satisfacen un criterio previamente establecido son eliminadas.

El modelo específico estimado para leche es:

$\ln Y_{1}=\beta_{0}+\beta_{1} \ln X_{1}+\beta_{2} \ln X_{2}+\beta_{3} \ln X_{3}+\beta_{4} \ln X_{4}+\beta_{5} \ln X_{5}+\beta_{6} \ln X_{6}+\beta_{7} \ln X_{7}+\varepsilon$

El modelo específico estimado para carne es:

$\ln Y_{2}=\beta_{0}+\beta_{1} \ln X_{1}+\beta_{2} \ln X_{2}+\beta_{3} \ln X_{3}+\beta_{4} \ln X_{4}+\beta_{5} \ln X_{5}+\beta_{6} \ln X_{6}+\beta_{7} \ln X_{7}+\varepsilon$

Donde:

$\mathbf{Y}_{1}=$ producción de leche;

$\mathbf{Y}_{2}=$ becerros producidos en el año;

$\mathbf{X}_{1}=$ alimento concentrado utilizado en la unidad de producción $\mathrm{kg}_{\text {año }}{ }^{-1}$;

$\mathbf{X}_{2}=$ vacas en producción;

$\mathbf{X}_{\mathbf{3}}=$ hectáreas en pastoreo;

$\mathbf{X}_{\mathbf{4}}=$ forraje conservado utilizado en la unidad de producción $\mathrm{kg}$ año ${ }^{-1}$;

$\mathbf{X}_{\mathbf{5}}=$ mano de obra;

$\mathbf{X}_{\mathbf{6}}=$ sementales;

$\mathbf{X}_{\mathbf{7}}=$ costos de insumos de operación (electricidad, gasolina, diésel);

$\boldsymbol{\beta}_{\mathbf{i}}=$ parámetros a estimar $(\mathrm{i}=0,1, \ldots, 7)$;

$\boldsymbol{\varepsilon}=$ es el termino residual. 
Después de estimar la función Cobb-Douglas con las variables que mejor explican la producción de carne y leche, con los valores de los coeficientes de los insumos, se calculó la elasticidad de producción, rendimientos marginales y etapa de producción de cada insumo. De acuerdo a las propiedades de una función Cobb-Douglas, el valor del coeficiente de cada insumo es igual a la elasticidad de producción de este, si es mayor a 1 el insumo tiene rendimientos marginales crecientes y si es menor a 1 presenta rendimientos marginales decrecientes. Además la elasticidad de la producción de cada uno de los insumos indica la etapa de producción en la que se encuentra, si el valor de $\beta \mathrm{i}>1, \beta_{\mathrm{i}}<1, \beta_{\mathrm{i}}<0$ se encuentran en la etapa I, II y III respectivamente ${ }^{(11)}$. Por otra parte, con la sumatoria de los coeficientes de los insumos de la función de producción para leche y carne se identificó el tipo de rendimientos a escala que tienen las empresas pecuarias. También se calculó el producto marginal $\left(\mathrm{PMgX}_{\mathrm{i}}\right)$ y el valor del producto marginal $\left(\mathrm{VPMgX}_{\mathrm{i}}\right)$ de los insumos derivado de la fórmula de elasticidades, utilizando las medias del total de la producción de leche y de los insumos con las siguientes fórmulas ${ }^{(14)}$.

$$
\begin{gathered}
\mathrm{Ep}\left(\mathrm{b}_{1}\right)=\frac{\partial \mathrm{Y} / \mathrm{Y}}{\partial \mathrm{X}_{\mathrm{i}} / \mathrm{X}_{\mathrm{i}}}=\frac{\mathrm{X}_{\mathrm{i}} \partial \mathrm{Y}}{\mathrm{Y} \partial \mathrm{X}_{\mathrm{i}}}=\frac{\mathrm{PMgX}_{\mathrm{i}}}{\mathrm{PPX}_{\mathrm{I}}} \\
\mathrm{PMgX}_{\mathrm{i}}=\operatorname{Ep}\left(\mathrm{b}_{1}\right) * \mathrm{PPX}_{\mathrm{i}} \\
V P M g X_{i}=P M g X_{i} * P Y_{I}
\end{gathered}
$$

Donde,

$\mathrm{PMgX}_{\mathrm{i}}=$ Producto marginal del insumo $\mathrm{X}_{\mathrm{i}}$

$\operatorname{Ep}\left(b_{1}\right)=$ La elasticidad de $Y_{i}$

$\mathrm{Y}_{\mathrm{i}}=$ Media de la producción anual de leche o becerros

$\mathrm{X}_{\mathrm{i}}=$ Media del insumo utilizado

$\mathrm{VPMgX}_{\mathrm{i}}=$ Valor del producto marginal $\mathrm{X}_{\mathrm{i}}$

$\mathrm{PY}_{\mathrm{I}}=$ Precio unitario de $\mathrm{Y}_{\mathrm{i}}$

$\mathrm{PPX}_{\mathrm{i}}=$ Producto promedio del insumo $\mathrm{X}_{\mathrm{i}}$ utilizado

El producto promedio de cada uno de los insumos fue el cociente entre la media de la producción (leche, becerros) y la media de los insumos (alimento, vacas).

El óptimo técnico de la producción de leche y carne se estimó por el método de multiplicador de Lagrange, optimizando la función de producción de leche y carne (funciones objetivo), sujeta a los precios de los insumos utilizados y precio de venta del producto (un litro de leche y un becerro). 


$$
L=f\left(X_{1}, X_{2}\right)-\lambda\left(P X_{1}+P X_{2}+M\right)
$$

Donde,

$\mathrm{L}=$ Función de Lagrange

$\lambda=$ Multiplicador de Lagrange

$\mathrm{F}\left(\mathrm{X}_{1}, \mathrm{X}_{2}\right)=$ Función de producción Cobb-Douglas para leche y carne

$\mathrm{PX}_{1}, \mathrm{PX}_{2}=$ Precio de los insumos variables

$\mathrm{M}=$ Precio unitario de producto

El procedimiento algebraico consistió en restar a la función objetivo la restricción, y se derivó parcialmente $\mathrm{L}$ (condición de primer orden) respecto a $\mathrm{X}_{1}, \mathrm{X}_{2}, \mathrm{y} \lambda$. Posteriormente por la regla de maximización, se igualó la relación de las derivadas parciales respecto a $\mathrm{X}_{1}, \mathrm{X}_{2}$ restringidos a la relación de precios de los insumos. Finalmente, se despejó y se obtuvieron los valores de $\mathrm{X}_{1}, \mathrm{X}_{2}$, los cuales se sustituyeron en la función Cobb-Douglas y se estimó el nivel óptimo técnico de producción de litros de leche y becerros.

El precio promedio de venta de un litro de leche y becerros fue de $\$ 5.38$ y $\$ 6020$ respectivamente. Mientras que el costo promedio de un $\mathrm{kg}$ de alimento fue de $\$ 4.0$ y el de una vaca productiva se estimó con la fórmula de recuperación de capital ${ }^{(15)}$. Donde el costo de compra de una vaquilla de remplazo fue de $\$ 18,000.00$ con una vida útil en el sistema DP de 8 años, y una taza de retorno anual del $12.5 \%$, el costo estimado de una vaca por año fue de \$500.00. Para el cálculo del óptimo técnico en la función de producción de leche se consideró el precio de una vaca por día, el cual fue el cociente del precio de una vaca por año/ 365 días, con un valor de $\$ 1.36$.

\section{Resultados y discusión}

La estructura de hato de las unidades de producción estudiadas se muestra en el Cuadro 1. Está estructura puede variar con la intensidad de producción y superficie disponible para realizar la actividad pecuaria dentro de las UP. Además de presentar constantes movimientos, debido al estado fisiológico de las vacas (vaquillas, secas, lactantes) o compra y venta de animales $^{(16)}$. 
Cuadro 1: Estructura del hato de unidades de producción en el sistema de doble propósito

\begin{tabular}{lllll}
\hline Variable & $\mathbf{n}$ & Media $(\overline{\boldsymbol{y}})$ & DE $(\mathbf{S})$ & $\mathbf{C V}$ \\
\hline Vacas en producción & 30 & 38.9 & 15.19 & 38.81 \\
Vacas secas & 30 & 18.97 & 9.91 & 52.24 \\
Vaquillas & 30 & 19.80 & 11,71 & 59.14 \\
Becerros & 30 & 14.22 & 8.54 & 60.05 \\
Becerras & 30 & 12.40 & 6.73 & 54.27 \\
Sementales & 30 & 2.49 & 1.49 & 59.83 \\
\multicolumn{1}{c}{$\mathrm{n}=$ Número de unidades de producción; DE= Desviación estándar; CV= Coeficiente de variación. }
\end{tabular}

En el modelo de la función de producción de leche y carne el coeficiente de determinación $\mathrm{R}^{2}$ indica que el $91.9 \%$ y $72.4 \%$ de la variabilidad de la producción es explicada por las variables independientes; InAlimento y Invacas respectivamente (Cuadro 2). El porcentaje de la variación no explicada en ambos modelos puede ser atribuida a diferencias entre las unidades de producción como; prácticas de manejo realizadas en el hato, o condiciones ambientales. En sistemas de producción de leche se reportan que el insumo alimento explica en mayor porcentaje la producción de leche respecto a otros insumos ${ }^{(15,17)}$. El uso de forraje verde, forraje conservado y alimentos concentrados pueden ser usados estratégicamente para aumentar la producción de leche ${ }^{(5,18)}$. Por lo tanto, el manejo y calidad de los alimentos es importante en los sistemas de producción de lechera, ya que está directamente relacionado con la producción.

En el Cuadro 3, se observa las medias de las variables productivas (leche y becerros) e insumos utilizados para la producción. En promedio con un hato de 39 vacas en producción, se obtiene una producción media anual de 93,678.5 L de leche y 14 becerros. 
Cuadro 2: Modelos de regresión seleccionados para producción de leche y carne

\begin{tabular}{llll}
\hline & $\begin{array}{l}\text { Parámetro } \\
\text { estimado }\end{array}$ & Error estándar & Pr $>\mathbf{F}$ \\
\hline Leche & & \\
\hline Intercepto & 6.099 & 0.310 & $<.0001$ \\
$\mathrm{InX}_{1}$ & 0.346 & 0.034 & $<.0001$ \\
$\mathrm{In} \mathrm{X}_{2}$ & 0.542 & 0.095 & $<.0001$ \\
$\mathrm{R}^{2}$ & 0.919 & & \\
\hline$\sum b_{i}=0.888$ & & & \\
\hline Carne & & 0.0579 \\
\hline Intercepto & 0.853 & 0.430 & $<.0001$ \\
InX & -0.205 & 0.047 & \\
In $\mathrm{X}_{2}$ & 1.118 & 0.133 & \\
$\mathrm{R}^{2}$ & 0.724 & & \\
$\sum b_{i}=0.913$ & & & \\
\hline InX $\mathrm{X}_{1}=$ logaritmo neperiano de kilogramos de alimento concentrado; In $\mathrm{X}_{2}=$ logaritmo neperiano de vacas en
\end{tabular}

Cuadro 3: Medias de producción anual de leche, becerros e insumos utilizados en unidades de producción en el sistema de doble propósito

\begin{tabular}{lllllll}
\hline Función & MPL & MPB & MIA & MIV & PPA & PPV \\
\hline Leche & $93,678.5$ & --------- & $45,678.9$ & 38.9 & 2.05 & $2,408.18$ \\
Carne & --------- & 14.22 & $45,678.9$ & 38.9 & $3.11 \times 10^{-04}$ & 0.365 \\
\hline
\end{tabular}

$\mathrm{MPL}=$ media de la producción de leche $\mathrm{kg}$ up año-1; MPB= media de la producción de becerros cabezas up año-1; MIA= media de insumo alimento $\mathrm{kg}$ up año ${ }^{-1}$; MIA= media de insumo vaca cabezas up año-1; PPA= producto promedio de insumo alimento; $\mathrm{PPV}=$ producto promedio de insumo vaca

\section{Función de producción Cobb-Douglas para leche}

$\operatorname{In} Y_{1}=6.099+0.346 \operatorname{In} X_{1}+0.542 \operatorname{In} X_{2}($ Ecuación 1$)$

Y por la trasformación, mediante la aplicación de antilogaritmos queda:

Leche $=e^{6.099} X_{1}^{0.346} X_{2}{ }^{0.542}$ (Ecuación 2)

Leche $=445.69 X_{1}{ }^{0.346} X_{2}{ }^{0.542}$ (Ecuación 3) 


\section{Elasticidades de producción para leche}

Es este estudio se encontró que aumentar en un $1 \%$ el insumo $\mathrm{X}_{1}$ (alimento), la producción total de leche aumentará en un $0.34 \%$, permaneciendo todos los demás insumos constantes. Mientras que un incremento del $1 \%$ en el insumo $\mathrm{X}_{2}$ (vacas), la producción total de leche aumentará en un $0.54 \%$. El incremento del uso de los insumos tiene un efecto positivo en la producción de leche, sin embargo, aumentar del número de vacas tendrá una mejor respuesta sobre la producción respecto al alimento. Este mismo comportamiento se encontró en otros sistemas de producción lechera en climas tropicales similares al área de estudio, quienes reportan que el insumo vacas presentó una mayor elasticidad de sus insumos utilizados, con valores de 0.40 a $0.60 \%$, seguido del insumo alimento con elasticidades de 0.15 a $0.30 \%$ (14) $^{(14}$. En el análisis de las empresas pecuarias, conocer el grado en que los insumos impactan la producción es importante, ya que se puede dar el caso en que las elasticidades de estos sean negativas e incrementar el uso de insumos, disminuyan la producción ${ }^{(10)}$, tal es el caso le lecherías tropicales de la India donde reportan una disminución del $2.4 \%$ en la producción de leche, al aumentar el consumo de concentrados ${ }^{(19)}$, lo cual trae perdidas económicas a la empresa pecuaria.

\section{Rendimientos marginales y etapa de producción de los insumos para producción de leche}

Los coeficientes de elasticidades de la función de producción de leche 0.34 para alimento y 0.54 para vacas (Ecuación 1). De acuerdo a la ley de los rendimientos marginales ambos insumos presentan rendimientos marginales decrecientes por ser valores menores a 1, y que se encuentran en la etapa II de una función de producción clásica, lo que significa que aumentar el uso de estos insumos, la producción de leche incrementará, pero en un menor grado conforme se adicionen estos insumos, hasta que la producción sea constante o comience a disminuir y llegue a la etapa III de la producción ${ }^{(10)}$. Aumentar la disponibilidad de alimentos a las vacas en un inicio aumentará la producción de leche, sin embargo, habrá un momento en que el animal alcance un máximo en su eficiencia alimenticia, es decir la cantidad de alimento en kilogramos que requiere el animal para producir un litro de leche ${ }^{(20)}$ y su metabolismo no sea capaz de absorber todos los nutrientes del alimento para aumentar la producción de leche y estos sean desechado por medio de la orina y heces, lo que representaría perdidas económicas para la empresa pecuaria por realizar un gasto en alimento, que el animal ya no está utilizando para la producción. Por otra parte, aumentar el número de vacas, manteniendo todos los demás factores constantes, reduciría la disponibilidad de recursos para estas, tales como la disponibilidad de alimento, teniendo como consecuencia una disminución en la producción total de leche. 


\section{Rendimientos a escala para producción de leche}

La función homogénea de producción de leche, presentó rendimientos decrecientes a escala, ya que la sumatoria de los coeficientes $\beta_{1}$ y $\beta_{2}$ fue de 0.888 , por lo tanto, un incremento porcentual similar en todos los insumos, determinará un aumento porcentual de menor magnitud en el producto obtenido ${ }^{(11)}$. Resultados similares han sido reportados en sistemas de producción de leche en el estado de Sinaloa ${ }^{(7)}$ donde este efecto es atribuido a una sobre utilización de los recursos que poseen la empresas pecuarias y faltas de uso de tecnología en el sistema, ya que empresas pecuarias grandes tienen rendimientos a escala crecientes, debido a la especialización del capital y trabajo ${ }^{(8)}$. Cuando se tiene este tipo de rendimiento a escala en las UP del sistema de DP, se debe evaluar el uso de los insumos, puesto que realizar un gasto en el incremento de estos, no traerá mayores ingresos por un aumento de la producción en la empresa pecuaria ${ }^{(21)}$, sino tendrán perdidas económicas por un gasto innecesario de insumos.

\section{Producto y valor del producto marginal para producción de leche}

El producto marginal para la producción de leche del insumo alimento indica que agregar 1 $\mathrm{kg}$ de este insumo, aumentará la producción de leche en $0.75 \mathrm{~L}$, con un ingreso adicional de $\$ 4.03$ por unidad de insumo adicionada, manteniendo los demás factores constantes. Mientras que aumentar una vaca en el hato, aumentará la producción de leche en $892.2 \mathrm{~L}$ al año, con un ingreso adicional de $\$ 4800.20$. En el sistema de DP en el estado de Sinaloa se han reportado valores del producto marginal mayores a cero del insumo vacas con rendimientos marginales decrecientes ${ }^{(7)}$, similares a este estudio, lo que significa que aumentar el tamaño del hato para incrementar la producción de leche, no es la mejor opción para aumentar la eficiencia en estos sistemas, sino más bien, hacer un uso óptimo de los insumos que mayor impacto tienen en la producción. Esto se complementa con el valor de producto marginal del insumo alimento y vacas obtenidos en este estudio, aunque ambos tienen un beneficio económico positivo en las empresas pecuarias, estos valores son menores a 1 , y de acuerdo a la ley de los rendimientos marginales, estos son clasificados como; rendimientos marginales decrecientes, y se ubican en la etapa II de una función de producción clásica, por tanto seguir aumentado unidades adicionales de estos insumos, provocará que el producto marginal continúe disminuyendo, hasta llegar a cero y hacerse negativo, provocando pérdidas económicas, tal es el caso de unidades de producción en la zona oriente de estado de Yucatán y sistemas de producción lechera tropical en la India, que tienen rendimientos marginales negativos para el insumo alimento y aunque los valores de producto marginal aun sigan siendo positivos, la producción de leche ya no aumenta ${ }^{(5,9)}$. 


\section{Óptimo técnico para la producción de leche}

$Y_{1}=445.69 X_{1}^{0.346} X_{2}^{0.542}$ sujeta a $4.0 X_{1}+1.36 X_{2}=5.38$

Por el método de Lagrange:

$L=445.69 X_{1}^{0.346} X_{2}^{0.542}-\lambda\left(4.0 X_{1}+1.36 X_{2}-5.38\right)$

La derivada parcial de $\mathrm{L}$ respecto a $\mathrm{X}_{1}$ y $\mathrm{X}_{2}$, bajo la condición de primer orden:

$4.0 \lambda=154.257 X_{1}^{-0.653} X_{2}^{0.542}$

$1.36 \lambda=241.64 X_{1}^{0.346} X_{2}^{-0.457}$

Igualación de las derivadas parciales respecto a $\mathrm{X}_{1}$ y $\mathrm{X}_{2}$ y sustituyendo $\mathrm{X}_{2}$ en la ecuación de restricción se obtiene la cantidad óptima de este insumo

$\frac{154.257 X_{1}^{-0.653} X_{2}^{0.542}}{241.64 X_{1}^{0.346} X_{2}^{-0.457}}=\frac{4.0}{1.36}$

$X_{2}=\frac{4.0 X_{1}}{0.229}=17.41 X_{1}$

$X_{1}=\frac{5.38}{27.677}=0.19$

$X_{2}=17.41(0.19)=3.38$

Sustituyendo los valores de $\mathrm{X}_{1}$ y $\mathrm{X}_{2}$ en la función Cobb-Douglas se obtiene la cantidad de leche óptima producida.

$Y_{1}=445.69(0.19)^{0.346}\left(3.38^{0.542}\right)=488.97 l$

Las empresas pecuarias del área de estudio alcanzan una producción óptima de leche de $488.97 \mathrm{~L}$ diarios, lo que equivale a que por vaca produzca $12.53 \mathrm{~L}$ diarios, ya que en promedio las empresas pecuarias tienen 40 vacas en producción. En los sistemas semi intensivos las vacas deben producir 35.38 L diarios para alcanzar la producción de leche óptima combinado los insumos alimento concentrado y forraje ${ }^{(22)}$, esta diferencia se debe principalmente a que reciben una mayor cantidad de concentrado respecto a los sistemas de producción del doble propósito. 


\section{Función de producción Cobb-Douglas para carne}

$\operatorname{In} Y_{2}=0.85366-0.20523 \operatorname{In} X_{1}+1.11829 \operatorname{In} X_{2}($ Ecuación 4)

Y por la trasformación, mediante la aplicación de antilogaritmos queda:

$Y_{2}=e^{0.85366} X_{1}^{-0.20523} X_{2}{ }^{1.11829}$ (Ecuación 5)

$Y_{2}=2.348 X_{1}{ }^{-0.20523} X_{2}{ }^{1.11829}$ (Ecuación 6)

\section{Elasticidades de producción para carne}

La función de producción de carne (Ecuación 6) muestra que un cambio del $1 \%$ en el número de vacas aumentará en un $1.11 \%$ la producción de becerros, mientras que el mismo cambio en la alimentación la disminuirá en un $0.20 \%$ manteniendo los demás factores constantes. En el sistema de doble propósito la alimentación de los becerros se basa en la lactancia controlada, que consiste en el consumo de leche de un cuarto de la ubre que se deja al becerro al momento de la ordeña y en la cantidad y calidad de forraje que consume en las praderas ${ }^{(23)}$. En este sistema la complementación alimenticia de los becerros con dietas de buena calidad, aunque mejoran el peso al destete, no hay diferencias en los precios pagados al productor, de esta forma los productores buscan alternativas para reducir el periodo al destete mediante la suplementación con forrajes alternativos (árboles y arbustivas forrajeras) que mejoren su desarrollo pre y post destete de los becerros ${ }^{(24)}$. Los resultados en este estudio muestran que la cantidad de alimento concentrado destinada al consumo de los becerros debe ser reducida ya que está no favorece la producción total de estos. En general el uso de alimento concentrado aumenta las variables productivas en los sistemas de producción ${ }^{(25)}$, sin embargo, la mejora dependerá de la cantidad y calidad del alimento, ya que, no conocer la cantidad de alimento necesaria a utilizar, se tendrán perdidas económicas de la empresa pecuaria por hacer un gasto innecesario.

\section{Rendimientos marginales y etapa de producción de los insumos para producción de carne}

El coeficiente de elasticidades para la función de producción de carne es negativo para el insumo alimento $\left(\mathrm{X}_{1}\right)$, mientras que para el insumo vacas es mayor a uno $\left(\mathrm{X}_{2}\right)$, (Ecuación 4). Valores mayores a uno, indica rendimientos marginales crecientes y que el insumo se encuentra en la etapa I de una función de producción clásica ${ }^{(10)}$, por tanto, aumentar el insumo vacas aumentará la producción de leche y sería poco sensato por parte del productor detener la producción en esta etapa. El mismo comportamiento se reporta en sistemas basados en 
pastoreo en el estado de México y Yucatán ${ }^{(8,9)}$ por lo tanto, aumentar el tamaño del hato con los recursos que cuentan estas unidades de producción se puede incrementar el rendimiento de las variables productivas. El alimento muestra el efecto contrario al tener una elasticidad menor a cero, estos significan rendimientos marginales negativos y que el insumo se encuentra en la etapa $\mathrm{III}^{(10)}$, es decir, aumentar la cantidad de alimento no beneficia la producción de becerros, al contrario, podría disminuirla. Efecto similar se reporta para la producción de carne en Yucatán, donde aumentar la cantidad alimento concentrado no mejora la producción de carne, por lo tanto, para aumentar el peso al destete en los becerros es mejor hacer un manejo adecuado de las praderas con las que se cuenta, para tener forrajes de buena calidad que cubran requerimientos nutricionales de los animales. Por otra parte, al ubicarse el insumo alimento en la etapa III de producción, a la empresa pecuaria no le es económicamente viable, porque está gastando dinero en un insumo que no aumenta sus ingresos por incrementos en la producción.

\section{Rendimientos a escala para producción de carne}

Las funciones de producción de carne muestran rendimientos decrecientes a escala debido a que la $\sum b 1$ es menor a uno. Es decir, aumentar todos los insumos en la misma proporción no aumentará la producción total, un efecto similar es reportado en empresas a pequeña y mediana escala en el Estado de México ${ }^{(8)}$. Mientras que las grandes empresas pecuarias, tienen rendimientos crecientes a escala, mismos que han logrado por medio de la mejora genética (capital) y eficiencia en el manejo de la empresa (trabajo) ${ }^{(8)}$. Por lo tanto, una alternativa para mejorar los rendimientos a escala para las pequeñas y medianas empresas pecuarias en el DP, sería aumentar la adopción de tecnología en el sistema.

\section{Producto y valor del producto marginal para producción de carne}

El producto marginal del insumo alimento para la producción de carne por ser menor a cero (Cuadro 4) indica que la producción total de becerros ya no aumentará, se ha alcanzado la producción máxima con una cantidad menor de alimento. Este insumo se está utilizando de forma excesiva y se tienen pérdidas de 0.38 centavos por cada unidad de alimento que se adiciona. Un efecto contrario se muestra con el número de vacas en producción, aumentar una unidad más este insumo tiene un ingreso de $\$ 2,460.93$ y por estar en la etapa I de la función de producción, el producto marginal seguirá incrementando y por tanto también su valor. El mismo comportamiento se reporta en unidades de producción del estado de Yucatán, quienes, al aumentar una unidad animal, incrementan la producción de carne a $980.7 \mathrm{~kg}, \mathrm{y}$ atribuyen que no han alcanzado su máxima producción, por la escasa utilización de programas de reproducción y mejoramiento genético ${ }^{(9)}$. Por lo tanto, a los productores en este estudio no les es convienente detener la producción de becerros con el número de vacas que tienen, podrían aumentarlas hasta que se encuentre en la etapa II de producción. 
Cuadro 4: Producto marginal y valor del producto marginal de insumos utilizados en la producción de leche y carne en el sistema de doble propósito

\begin{tabular}{|c|c|c|c|c|c|c|}
\hline \multirow{2}{*}{ Función } & \multicolumn{2}{|c|}{ Precio unitario \$ } & \multicolumn{2}{|l|}{ Alimento } & \multicolumn{2}{|c|}{ Vacas } \\
\hline & Leche & Becerros & PMg & VPMg & PMg & VPMg \\
\hline Leche & 5.38 & ---------- & 0.75 & 4.03 & 892.2 & 4800.2 \\
\hline Carne & ------- & 6020 & $-6.38 \mathrm{exp}^{-05}$ & -0.38 & 0.408 & 2460.93 \\
\hline
\end{tabular}

\section{Óptimo técnico para la producción de carne}

$Y_{2}=2.34 X_{1}^{-0.205} X_{2}^{1.118}$ sujeta a $4.0 X_{1}+500 X_{2}=6,020$

Por el método de Lagrange:

$L=X_{1}^{-0.205} X_{2}^{1.118}-\lambda\left(4.0 X_{1}+500 X_{2}-6,020\right)$

La derivada parcial de $\mathrm{L}$ respecto a $\mathrm{X}_{1} \mathrm{y} \mathrm{X}_{2}$, bajo la condición de primer orden:

$4.0 \lambda=0.481 X_{1}^{-1.205} X_{2}^{1.188}$

$500 \lambda=2.625 X_{1}^{-0.205} X_{2}^{-0.188}$

Igualación de las derivadas parciales respecto a $\mathrm{X}_{1}$ y $\mathrm{X}_{2}$

$\frac{0.481 X_{1}^{-1.205} X_{2}^{1.188}}{2.625 X_{1}^{-0.205} X_{2}^{-0.188}}=\frac{4.0}{500}$

$X_{2}=\frac{4.0 X_{1}}{91.61}=0.043 X_{1}$

Sustituyendo $\mathrm{X}_{2}$ en la ecuación de restricción se obtiene la cantidad óptima de este insumo y los valores de $\mathrm{X}_{1}$ y $\mathrm{X}_{2}$ en la función Cobb-Douglas se obtiene la cantidad de leche óptima producida.

$X_{1}=\frac{6,020}{25.5}=236.07$

$X_{2}=0.043(233.07)=10.151$

$Y_{2}=2.348(236.07)^{-0.205}(10.151)^{1.118}=10.22$ becerros

El óptimo técnico para la producción de carne en el área de estudio es 10.22 becerros al año. Con una combinación de insumos $\mathrm{X}_{1}=236.07$ y $\mathrm{X}_{2}=10.15$ que maximizan la isocuanta de producción de becerros. 


\section{Conclusiones e implicaciones}

Los insumos alimento y número de vacas son los insumos que mejor explican la producción de leche y carne en las empresas pecuarias de doble propósito en las áreas de estudio, por lo tanto, los productores deben poner atención en el uso de estos insumos, ya que un uso irracional puede disminuir las variables productivas. Por otra parte, las elasticidades de producción indican que un aumento de los insumos utilizados para la producción de leche y carne, incrementan la producción total manteniendo todos los demás factores constantes, a excepción del insumo alimento para la producción de carne. Los insumos alimento y vacas para la producción de leche presentan rendimientos decrecientes a escala, y se encuentran en la etapa II de la producción, etapa en la que se recomienda que la empresa pecuaria produzca, ya que en el caso de la producción de carne, el insumo alimento se ubicó en la etapa III de una función de producción, porque el valor del producto marginal fue negativo lo que ocasiona pérdidas económicas para la empresa y a esta no le es conveniente operar en esta etapa de producción. Aunque los insumos para la producción de leche tienen un valor del producto marginal positivo, estos no deben ser incrementados, ya que se encuentran operando en la ley de los rendimientos marginales decrecientes. Por otra parte, debido a que las empresas pecuarias presentan rendimientos decrecientes a escala, una alternativa para mejorar estos rendimientos podría ser aumentar el uso de tecnología en diferentes áreas (alimentación, manejo de praderas, conservación de forrajes, reproductivas, capacitación técnica) con la finalidad de especializar el capital y el trabajo en estos sistemas.

\section{Literatura citada:}

1. SIAP. Servicio de Información Agroalimentaria y Pesquera. Resumen Nacional Pecuario. 2018. México.

2. Rojo RR, Vázquez AJF, Pérez P, Mendoza MGD, Salem AZM, Albarrán PB, et al. Dualpurpose cattle production in Mexico. Trop Anim Health Prod 2009;41(5):715-721.

3. Orantes ZMÁ, Platas RD, Córdova AV, Santos LD, Carmen M, Córdova AA. Caracterización de la ganadería de doble propósito en una región de Chiapas, México. Ecosistemas y recursos agropecuarios 2014;1(1):49-58.

4. Albarrán PB, Rebollar RS, García MA, Rojo RR, Avilés NF, Arriaga JCM. Socioeconomic and productive characterization of dual-purpose farms oriented to milk production in a subtropical region of Mexico. Trop Anim Health Prod 2015; 47(3):519-523.

5. Vishnoi S, Pramendra, Gupta V, Pooniya R. Milk production function and resource use efficiency in Jaipur District of Rajasthan. Afr J Agric Res 2015;10-32:3200-3205. 
6. Bravo UBE, Rieger L. Alternative production frontier methodologies and dairy farm efficiency. JAE 1990;41(2):215-26.

7. Cuevas RV, Loiza MA, Astego CH, Moreno GT, Borja BM, Reyes JJE, et al. Análisis de la función de producción de leche en el sistema bovinos doble propósito en Ahome, Sinaloa. Rev Mex Cienc Pecu 2018;9(2):376-386.

8. Morales HJL, Gonzales RFDJ, Hernández MJ. Función de producción de la ganadería de carne en la zona sur del Estado de México. Rev Mex Cienc Pecu 2018;9(1):1-13.

9. Pech MV, Santos FJ, Montes PR. Función de producción de la ganadería de doble propósito de la zona oriente del estado de Yucatán, México. Téc Pecu Méx 2002;40(2):187-192.

10. Debertin DL. Agricultural production economics. $2^{\text {nd }}$ ed. New York, USA: MacMillan; 1982.

11. Vargas BBE. La Función de producción Cobb - Douglas. Revista de Difusión Cultural y Científica de la Universidad La Salle en Bolivia 2014;8(8):67-74.

12. CNA. Comisión nacional del agua. Normales climatológicas. México; 2017.

13. Griliches Z. Estimates of the Aggregate Agricultural Production Function from crosssectional data. J Farm Econom 1963;45(2):419-28.

14. Ghebremariam WK, Ortmann GF, Nsahlai IV. A production function analysis of commercial dairy farms in the Highlands of Eritrea using ridge regression. JAE 2006; 45(2):225-241.

15. Monke EA, Pearson SR. The policy analysis matrix for agricultural development. Policy Anal; 1989.

16. Quiroz J, Granados L, Barrón M, Espejel A, Espinosa JA. Estructura de los hatos bovinos en Tabasco. AICA 2014;(4):252-253.

17. Mahajan S. Economic analysis of rural and pere urban farm in Ludhiana District of Punjab [M.Sc.Thesis. Karnal Haryana, India. Deemed University; 2008.

18. Sinhg S. Economic analysis of milk production in Varanasi District of Utta Pradesh. [M.Sc.Thesis].Karnal, Haryana, India: Deemed University; 2008.

19. Ashish C, Bhadauria A. Production function analysis on member dairy cooperative society for milch cow in Distric Etawah (U.P). IJEAB 2017; 2(1):23-29.

20. Mackle TR, Parr CR, Stakelum GK, Bryant AM, MacMillan KL. Feed conversion efficiency, daily pasture intake, and milk production of primiparous Friesian and Jersey 
cows calved at two different liveweights. N Z J Agric Res 1996; 39(3):357-70.

21. Cursack AM, Castignani MI, Osan O, Castignani H. Función de producción en sistemas lecheros de alta producción de la Cuenca Central Santafesina, Argentina. $11^{\circ}$ Congreso Panamericano de la Leche. Bello Horizonte, Brasil; 2010.

22. Rebollar RS. La función Cobb-Douglas de la producción semi -intensiva de leche en el sur del Estado de México. Análisis económico. Repositorio Institucional UNAM. 2018;(82) vol. XXXIII.

23. Pérez HP, Becerril PCM, Lamothe ZC, Torres HG, López OS, Gallegos SJ. Efecto del amamantamiento retrasado en la actividad posparto de las vacas y en los becerros de doble propósito. Interciencia 2006;31:748-752.

24. Pérez HP, Solarís MF, García WM, Osorio AM. Gallegos SJ. Comportamiento productivo y reproductivo de vacas de doble propósito en dos sistemas de amamantamiento en trópico. Arch Latinoam Prod Anim 2001;9:79-85.

25. Camacho VJH, Cervantes EF, Palacios RMI, Rosales NF, Vargas CJM. Factores determinantes del rendimiento en unidades de producción de lechería familiar. Rev Mex Cienc Pecu 2017;8(1):23-29. 